\title{
A Re-usable Biosensor for Organophosphate Pesticides
}

\author{
Shinji OKAZAKI*, Hidemoto NAKAGAWA, Shukuji ASAKURA, Kenzo FUKUdA, \\ Hiromi KIUCHI $^{\dagger}$, Sachio TAKAHASHI ${ }^{\dagger}$ and Tesshi SHIGEMORI ${ }^{\dagger}$
}

Received December 26, 1997 ; Accepted March 20, 1998

\begin{abstract}
An enzyme sensor for the detection of organophosphate pesticides in water was fabricated. Acetylcholinesterase and cholineoxidase were co-immobilized on a Pt electrode by crosslinking. Enzymatic activity was measured amperometrically by the generation of $\mathrm{H}_{2} \mathrm{O}_{2}$. Dichlorvos (DDVP, dimethyl 2,2dichlorovynyl phosphate) was chosen as a model pesticide and its inhibition characteristics were utilized for the pesticide detection. A good linear correlation was observed between the Relative Inhibition (RI) and pesticide concentrations with high sensitivity. The re-activation characteristics of inhibited enzyme by 2-pyridinealdoxime methochloride (PAM) were investigated in detail. It has been found that the enzyme electrode may be used several times for the inhibition detection if proper recovery procedure is enforced. The excessive treatment by PAM, however, deteriorates the immobilized enzyme. The result indicates that the sensor is highly promising for the continuous monitoring of organophosphates.
\end{abstract}

\section{INTRODUCTION}

Although agricultural application of organophosphate pesticides benefited us tremendously in food productions, they are toxic to human beings as well. Since there is a case of pesticides' contamination to drinking water and agricultural products, a method to detect these pesticides with high sensitivity is desired. A GC/MS (gas chromatography/mass spectrometry) satisfies this demand and more than 100 pesticides can be analyzed with the identifications of individual chemicals. ${ }^{1)}$ However, the method requires elaborate and cumbersome procedures and only intermittent analysis of every 30 minutes can be performed.

An enzymatic method that utilizes the inhibition action of pesticides to cholinesterase has been suggested. ${ }^{2)}$ Although the method cannot distinguish individual chemical species, it is believed to be a good measure for the toxicity to the human because it simulates the immediate toxic action of pesticides. ${ }^{3-7)}$ It is suitable for continuous monitoring of drinking waters, ${ }^{3,4)}$ and can detect toxicity in a few minutes if

Department of Material Science and Chemical Engineering, Faculty of Engineering, Yokohama National University (79-5, Tokiwadai, Hodogaya, Yokohama, 240-8501, JAPAN)

${ }^{\dagger}$ New Cosmos Electric Co. Ltd. (2-4-5, Mitsuya-Naka, Yodogawa, Osaka 532-0036, JAPAN)

Key Words : Biosensor, Organophosphate Pesticide, DDVP, PAM the pesticide concentration is high. It is also applicable to a field investigation because of a small system with less power consumption. These characteristics should be contrasted with the GC/MS method. A GC/MS has very high sensitivity, but the system is heavy and bulky. About 30 minutes may be required to obtain results.

The enzymatic method, however, has some drawbacks of a long term instability and of a intrinsically disposable nature of the electrode once pesticides are detected. There are a few reports ${ }^{8-10)}$ concerning the reactivation of inhibited enzyme electrodes, but not all of them reported the successful recoveries and further investigation is desired to establish the re-activation technique. Biosensors of this type tend to show some variations in sensitivities, and often one has to calibrate the sensitivity for each device before use. But the irreversible nature of inhibition action makes calibration difficult. Therefore it is important for these sensors to be re-activated and to be re-usable. The research is focused on the re-activation of the cholinesterase immobilized on the Pt electrode by PAM.

\section{PRINCIPLE}

The mechanism of immediate biohazard action from organophosphate pesticides is the formation of irreversible compounds with acetylcholinesterase(AChE) and butyrylcholinesterase(BChE) by phosphorylating the serine in active site of the enzymes." DDVP was 
chosen as a model chemical for the pesticides in this study.

$$
\begin{gathered}
\text { Enz.-Ser-OH }+ \text { R-P-OR' } \\
\text { O' OR" } \\
\rightarrow \text { Enz--Ser-O-P-OR' + HR } \\
\text { O' OR" }
\end{gathered}
$$

In the absence of pesticides, AChE (BChE) hydrolyses acetylcholine (butyrylcholine) to acetic acid (butyric acid) and choline.

$$
\text { Acetylcholine }+\mathrm{H}_{2} \mathrm{OChE} \text { Choline }+ \text { Acetic acid }
$$

Acetylcholine is the neurotransmitter that transfers stimulus between synapses and malfunction of these enzymes results continuous stimulation of synapses and muscle. By supplying proper amount of acetylcholine (substrate) and measuring products of the reaction (2), decrease by enzymatic inhibition caused by pesticides, can be monitored. ${ }^{2 y}$ Several methods to measure enzymatic activities have been suggested. One of the product, acetic acid, leads to changes of $\mathrm{pH}$ and pesticides may be monitored by $\mathrm{pH}$ measurements. ${ }^{8,9)}$ If one uses acetylthiocholine instead of acetylcholine, thiocholine is produced which is easily detected by an anodic oxidation. ${ }^{6}$ ) Probably a more stable and versatile method of pesticide detection is the use of a second enzyme, Cholineoxidase ( $\mathrm{CO}){ }^{5.7,10)} \mathrm{CO}$ oxidizes choline to betaine and hydrogen peroxide.

$$
\text { Choline }+2 \mathrm{O}_{2}+\mathrm{H}_{2} \mathrm{O} \stackrel{\mathrm{CO}}{\rightarrow} \text { Betaine }+2 \mathrm{H}_{2} \mathrm{O}_{2}
$$

The resultant $\mathrm{H}_{2} \mathrm{O}_{2}$ can be easily oxidized by electrochemical reaction and oxidation current may be measured amperometrically for the enzyme activities. This method was adopted in the present research.

It may be convenient to define the Relative Inhibition (RI) parameter, as follows

$$
R I=\frac{(d i / d t)}{i_{s s}}
$$

Where $(d i / d t)$ : absolute rate of the current decrease $i_{s s}$ : steady state oxidation current.

It can be shown that RI is proportional to the inhibitor concentration when its concentration is low in comparison to substrate concentration. ${ }^{11)}$

PAM, which has stronger coupling ability to pesticides, has been adopted for the re-activation of inhibited AChE. It re-activates the enzyme by extracting phosphate according to the following reaction.

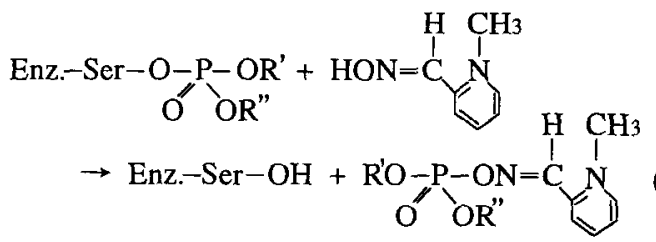

\section{EXPERIMENTAL}

Acetylcholinesterase (AChE, EC 3.1.1.7, 1000 $\mathrm{U} / \mathrm{mg}$, from Electric eel, type V-S) and cholineoxidase (CO, EC 3.1.1.17, $10 \mathrm{U} / \mathrm{mg}$, from Arthrobacter globiformis) were purchased from SIGMA Chemicals. Glutaraldehyde (70\% solution, for biochemistry), albumin (10\%, from bovine serum), acetylcholine chloride (analytical grade), choline chloride (analytical grade), and DDVP standard ( $99 \%$ purity) were obtained from Wako Chemicals. PAM (99\% purity) was purchased from Aldrich Chemicals. Co.

The enzyme electrode, shown in Fig. 1, was fabricated by the following procedures. About $29 \mathrm{U}$ of $\mathrm{AChE}$ and $58.5 \mathrm{U}$ of $\mathrm{CO}$ were dissolved in $100 \mu 1$ of phosphate buffer $(0.02 \mathrm{M})$ solution. $5 \mu 1$ of the enzyme solution was coated directly onto a $5 \mathrm{~mm} \phi \mathrm{Pt}$ plate which was attached to an acrylic cylinder and dried. Then $50 \mu 1$ of the above solution was mixed with $50 \mu 1$ of $10 \mathrm{wt} \%$ albumin and $1 \mu \mathrm{l}$ of $70 \mathrm{wt} \%$ glutaraldehyde. $5 \mu \mathrm{l}$ of this solution was coated onto the enzyme-coated Pt plate and dried to form a crosslinked enzyme layer.

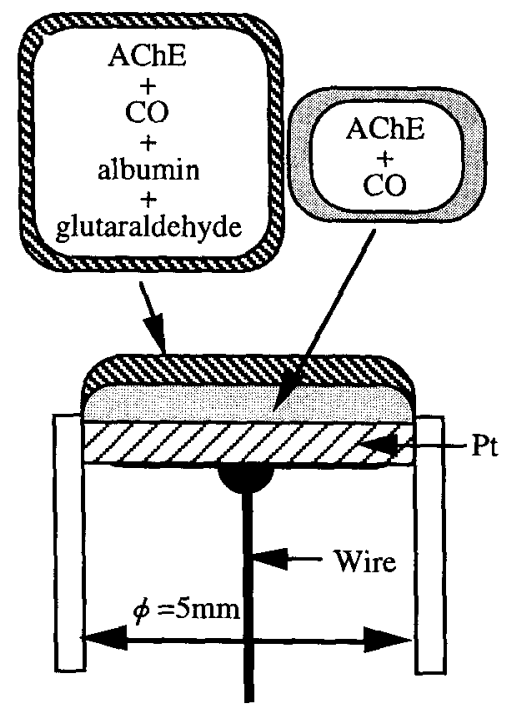

Fig.1 Illustrative cross-sectional view of the enzyme electrode. 
By placing the enzyme layer without albumin below the crosslinked layer, higher detection current is expected. Several electrodes were fabricated at a time, and about $2.9 \mathrm{U}$ of AChE and $5.85 \mathrm{U}$ of $\mathrm{CO}$ were immobilized on each electrode if no deterioration had occurred during the immobilization. The sensor was conditioned in the $0.02 \mathrm{M}$ phosphate buffer solution at least 24 hours before use.

The activity of enzyme was measured in the $0.02 \mathrm{M}$ phosphate buffer solutions of $\mathrm{pH}$ 6.8. The resultant $\mathrm{H}_{2} \mathrm{O}_{2}$ was measured amperometrically by a three electrode potentiometric system with an applied voltage of $600 \mathrm{mV}$ vs. $\mathrm{Ag} / \mathrm{AgCl}$ reference electrode. All measurements were performed at room temperature of about 25 ${ }^{\circ} \mathrm{C}$ without active temperature control.

\section{RESULTS AND DISCUSSION}

Figure 2 shows the relationship between acetylcholine concentration and oxidation current. In the low concentration range, the current is proportional to the concentration, but tends to saturate at above $2 \mathrm{mM}$. Saturated range should be used for the inhibitor detection, and substrate concentration was determined to be $3.5 \mathrm{mM}$ for the pesticide measurement.

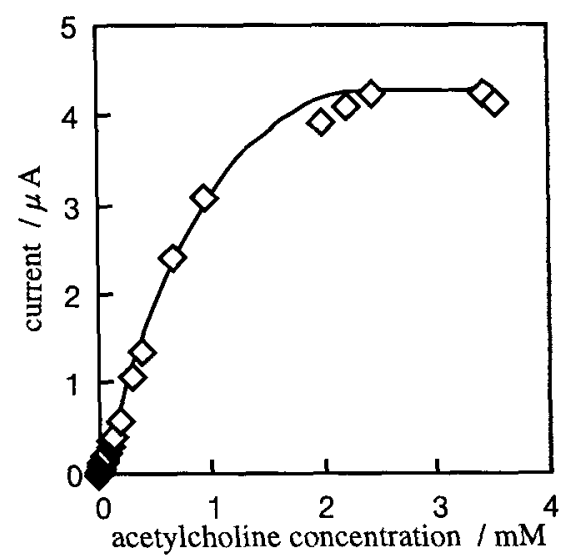

Fig.2 Relationship between the concentration of acetylcholine and the current.

When DDVP was added to the solution, oxidation current decreased almost exponentially. RI value may be obtained from the time dependence of the current decrease. (di/dt) was calculated from the initial slope of the decay curve. The current without background before the inhibition was taken as steady state current $i_{s s}$. RI values are plotted in Fig. 3. It is clear that RI increases linearly with the concentrations as theory predicts. Although the data were not shown, the good linear relationship holded up to $1.2 \mathrm{ppm}$ and started to saturate above this concentration. The sensitivity of the current sensor was very high despite the crude design. The lowest concentration we had tested was $46 \mathrm{ppb}$. This sensitivity would satisfy a requirement for environmental monitoring. Optimization in designing could further decrease the detection limit, but the investigation was concentrated to the re-activation. Hendji et al. reported the detection limit of $0.2 \mathrm{ppt}$ for DFP (diisopropyl fluorophosphate). ${ }^{\text {) }}$

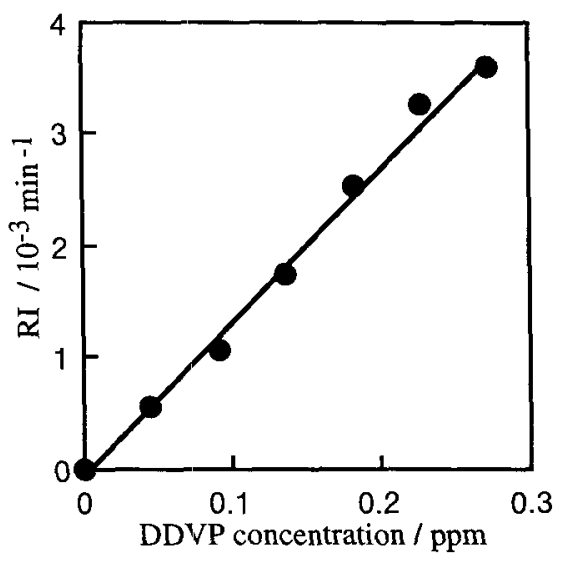

Fig. 3 Calibration curve for DDVP.

As stated in the introduction, RI values may be obtained as a result of irreversible reaction of $\mathrm{AChE}$ and DDVP with a sacrifice of enzyme activity. Establishment in re-activation technology is strongly desired for an accurate and reliable measurement. Figure 4 shows the current response of the sensor with the repeated exposures to $3.5 \mathrm{mM}$ substrate, $4.5 \mathrm{ppm}$ inhibitor (DDVP), $0.05 \mathrm{M}$ PAM in sequence. The DDVP concentration of $4.5 \mathrm{ppm}$ was chosen based on the expectation that the heavy contamination of more than 5 $\mathrm{ppm}$ is rather infrequent. Initially small background current of less than $0.5 \mu \mathrm{A}$ was flowing in the phosphate buffer solution. In $3.5 \mathrm{mM}$ substrate (acetylcholine) solution the current increased to more than $4 \mu \mathrm{A}$ due to the generation of $\mathrm{H}_{2} \mathrm{O}_{2}$. The sensor was kept in the substrate solution for few hours to check the stability. DDVP was added to constitute a concentration of $4.5 \mathrm{ppm}$ for the inhibition measurement. The sensor was left in the DDVP solution for 30 minutes. The current decreased to less than a half of its pre-inhibition value. The lowest point of the decreasing curve indicated the current after the 30 minutes inhibition, and the sensor was removed from the DDVP solution at this time. Then sensor was dipped in $0.05 \mathrm{M}$ PAM solution for 5 minutes and washed in a phosphate buffer solution for 24 hours. The sensor 
was placed in the phosphate solution and next sequence of the measurement was started. This sequence was repeated 5 times. Every time the sensor was exposed to DDVP, the current decrease was observed, and the substrate current increased to nearly its pre-inhibition value after the re-activation. The effect of PAM re-activation is clear and the recovery rate is close to $100 \%$. Slight decrease may be caused partly by the incomplete

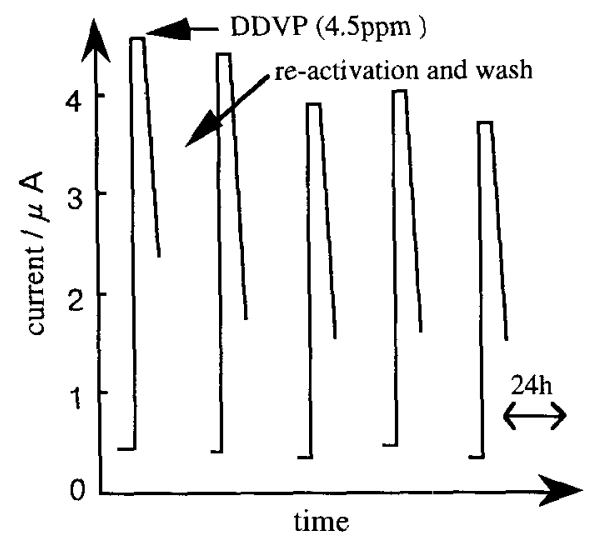

Fig.4 Inhibition and re-activation characteristics with $0.05 \mathrm{M}$ PAM.

re-activation. But loss of enzyme by thorough washing, and the natural degradation could be another reason. Similar experiment was performed with the DDVP concentration of $0.9 \mathrm{ppm}$ and exposure time of 2 hours. Good recovery rate of almost $100 \%$ was observed. When the sensor was exposed to high concentration( $>100 \mathrm{ppm}$ ) of DDVP, however, the recovery rate is poor with a value of some $50 \%$. Yamani et al. ${ }^{8)}$ reported similar phenomena with the inhibition by paraoxon and re-activation by PAM. They reported complete recovery with paraoxon concentration of less than $5 \times 10^{-7} \mathrm{M}$, but incomplete recovery at $2 \times 10^{-6} \mathrm{M}$ concentration. Hendji et al. ${ }^{9}$ reported that only partial recovery was obtained in their experiments with PAM. They claimed that, recovery rate had maximum value with exposure time of 30 minutes, and longer exposure resulted in poor recovery. They suspected a characteristic change of the enzyme membrane by a PAM treatment.

Re-activation of the inhibited enzyme was performed with different concentrations of PAM solutions to investigate the recovery phenomenon. The results are presented in Fig. 5. Inhibition and recovery procedures were identical to those of Fig. 4, except PAM concentrations. Different sensors were used for the different concentrations of PAM, and recovery rates were normalized by their original detection current. It should be mentioned that the sensors, fabricated simultaneously, showed relatively uniform detection characteristics. The variations in the steady currents of a same batch were less than $20 \%$. Although the recovery rate with 0.01 M PAM exceeded slightly $100 \%$, it is clear that recovery rate with $0.1 \mathrm{M}$ PAM decreased as it experienced inhibition and recovery processes. The sensitivity to the inhibitor of the sensor re-activated with 0.1 M PAM solution, decreased as exposure to the inhibitor and recovery processes were repeated. Whereas the other sensors showed constant or even increased sensitivities with the repeated exposures at lower concentrations.

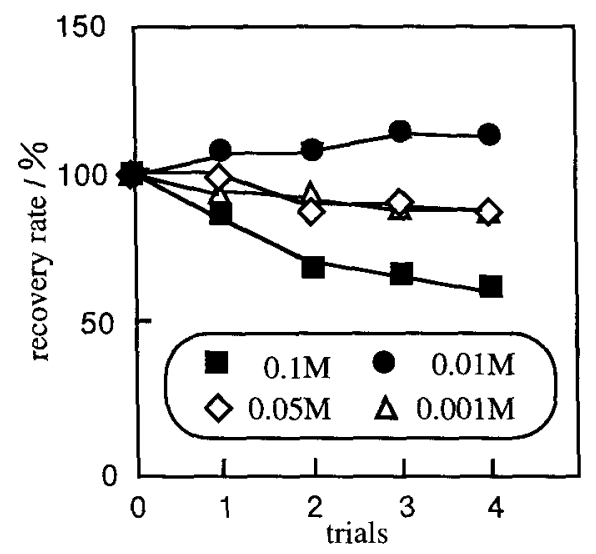

Fig.5 Effect of PAM concentrations on recovery.

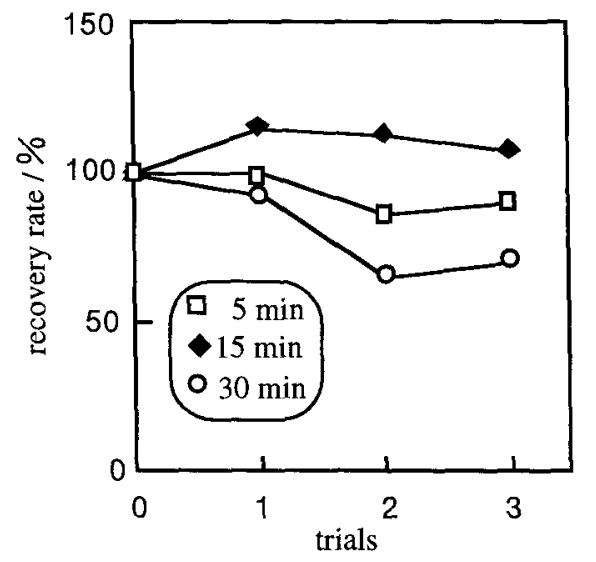

Fig.6 Effect of PAM treatment time on recovery.

Re-activation experiments by $0.05 \mathrm{M}$ PAM with different dipping times were also performed. Inhibition and recovery procedures were identical to those of Fig. 4 , except PAM treatment time. Figure 6 shows that the inhibition recovery rates were close to $100 \%$ if inhibited sensors were kept in the PAM solution less than $15 \mathrm{~min}$. But the recovery rate decreased if they 
were left in the solution more than $30 \mathrm{~min}$. Poor recovery rate of long time exposure to PAM is in agreement with Hendji et al. ${ }^{9)}$ as stated previously. The above observations suggest that PAM re-activates phosphate inhibited enzyme, yet dense or prolonged exposure damages the enzyme membrane permanently.

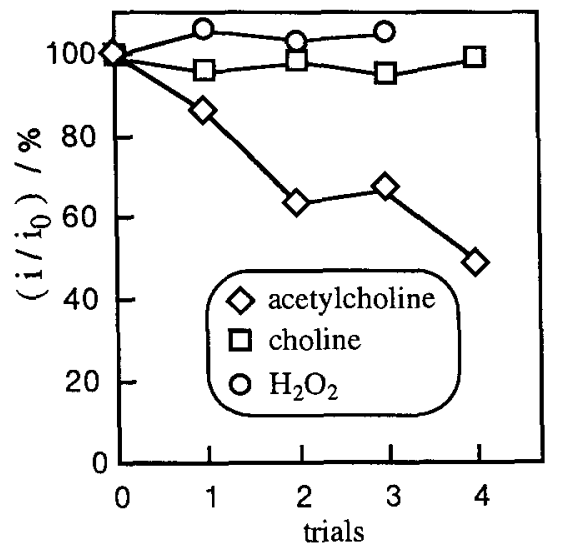

Fig. 7 Effect of PAM re-activations on currents by several sources.

To investigate the poor recovery phenomenon in the 0.1 M PAM solution, the currents after the recovery operation by different source materials, i.e. acetylcholine, choline, $\mathrm{H}_{2} \mathrm{O}_{2}$ were measured. Data were taken by the following procedures: First, the oxidation currents of a sensor due to $1 \mathrm{mM} \mathrm{H} \mathrm{H}_{2} \mathrm{O}_{2}, 5 \mathrm{mM}$ choline, 3.5 $\mathrm{mM}$ acetylcholine, all in phosphate buffer solutions were measured. Next the sensor was treated with $0.1 \mathrm{M}$ PAM for 5 minutes and washed in phosphate buffer and conditioned for about 24 hours. Then the oxidation currents were measured again. The treatments were repeated 4 times. The results were normalized and shown in Fig. 7. Since the currents by choline and by $\mathrm{H}_{2} \mathrm{O}_{2}$ after the recovery were always almost $100 \%$, it may be concluded that physical properties of the membrane and the activity of the immobilized CO had not changed. It is likely that immobilized AChE lost its activity by the PAM treatment. Since the sensor was not exposed to DDVP inhibition, the PAM treatment could be the sole origin for the loss of AChE activity.

\section{CONCLUSION}

The present research indicates that an AChE and CO co-immobilized enzyme electrode is suitable for the continuous monitoring of the toxic organophosphates. Although only the effect of DDVP was investigated, it has been known that AChE inhibited by various organophosphate pesticides and carbamates. ${ }^{4,6,8,11)}$

The recovery process of the inhibited enzyme electrode by PAM treatment was investigated in detail. It is shown that the effect of re-activation by PAM is relatively strong, and excessive treatment may result in deterioration of immobilized enzyme. The electrode may be used several times if the inhibition is not strong and the proper recovery procedure is enforced. Further investigation is necessary for the elucidation of poor recovery in the cases of strong inhibition and of strong recovery treatment.

\section{References}

1) T. Okumura and K. Imamura, Suishitsu odaku kenkyu, 14, 109(1991). In Japanese

2) E.K. Bauman, L.H. Goodson, G.G. Guilbault and D.N. Kramer, Anal.Chem., 37, 1378(1965).

3) L.H. Goodson and W.J. Jacob, "Methods in Enzymology" , 44, 647(1976),, Academic Press.

4) R. Kindervater, W. Kuennecke and R.D. Schmid, Anal. Chim. Acta, 234, 113(1991).

5) U. Wollenberger, K. Setz, F.W. Scheller, U. Loeffler, W. Goepel and R. Gruss, Sensors and Actuators $B, 4,257(1991)$.

6) P. Skladal, Anal. Chim. Acta, 252, 11(1991).

7) G. Palleschi, M. Bernabei, C. Cremisini and M. Mascini, Sensors and Actuators B., 7,513(1992).

8) H.El. Yamani, C. Tran-Minh, M.A. Abdul and D. Chavanne, Sensors and Actuators, 15, 193(1988).

9) A.M. Nyamsi Hendji, N. Jaffrezic-Renault,C.Martelet, P. Clechet, A.A. Shulga, V.I. Strikha, L.I. Netchiporuk, A.P. Soldatkin and W.B. Wlodarski, Anal. Chim. Acta, 281, 3(1993).

10) J-L. Marty, K. Sode and I. Karube, Electroanalysis, 4, 249(1992).

11) P. Skladal, Anal. Chim. Acta, 269, 281(1992). 